\title{
Selbstschilderung eines Falles von schizophrener Psychose.
}

\author{
Von \\ F. Schwab (Frankfurt a. d. O.). \\ Mit 2 Textabbildungen. \\ (Eingegangen am 1. Juli 1918.)
}

\section{Einleitung.}

Der Arzt befindet sich dem Geisteskranken gegenüber in einer ungünstigeren Lage als dem nur körperlich Kranken, wenn er sich bemüht, die subjektiven Empfindungen und Erlebnisse des Kranken zu verstehen. Mit verhältnismäßig einfachen Mitteln gelingt es ihm zwar, die Geisteskrankheit bald mit einem Namen zu nennen, sie zu klassifizieren; für die Erforschung eigenartiger Bewußtseinszustände sind bestimmtere Methoden erforderlich, z. B. die Einfühlung. Aber auch diese wird unzureichend, wo es sich $\mathrm{um}$ Zustände handelt, die weniger bekannt sind, deren Inhalte noch keinen Vergleich erlauben. Da sind zunächst Selbstdarstellungen wichtig, sie zichen weniger bekannte und weniger zugängliche Zustände ans Licht.

Geisteskranke können oft nur wenig oder gar keine Auskunft geben über ihren Zustand oder es besteht die Gefahr der unvollkommenen Darstellung und der Täuschung. Und geisteskrank gewesene Menschen sind selten in der Lage, ihren Zustand und den Verlauf der Krankheit objektiv zu betrachten und zu schildern.

Aber es gibt Ausnahmen. Ws gibt gute Selbstschilderungen; sie haben schon viel beigetragen zur Aufklärung über psychopathologische Fragen, über das Wesen der Halluzination, der Schizophrenie und der verschiedensten Psychosen und psychopathischen Zustände. Bei letzteren sind Selbstschilderungen um so wertvoller, weil sie nicht häufig zur Beobachtung in die Klinik kommen. Und Gebildete, die gute Selbstschilderungen geben könnten, bilden die kleinere Zahl der psychisch Erkrankten; oft sind sie auch nicht dlazı zu hewegen, ihren Fall veröffentlichen zu lassen.

Jas pers ${ }^{1}$ ) weist dringend auf die Wichtigkeit der Selbstdarstellung hin und fordert auf, solche Fälle zu veröffentlichen. Desgleichen betont Gruhle ${ }^{2}$ ) die Selbstschilderung gebildeter Geistcskranker. Aus diesen Gründen dürfte die Veröffentlichmng des vorliegenden Falles 
bei der Geringfügigkeit des bisher publizierten Materials besonders wertvoll sein.

Gute Selbstschilderungen waren schon von grundlegender Bedeutung; ich erinnere nur an die Darstellungen von Johannes Müller ${ }^{3}$ ) über Gesichtshalluzinationen, sowie vor allem an die Selbstschilderung des Psychiaters $\mathrm{Kandins} \mathrm{ky}^{4}$ ), der eine von ihm selbst durchgemachte Halluzinationspsychose analysierte. Im Anschluß an diese Veröffentlichung trat eine bedeutende Wandlung in den Anschauungen über das Wesen der Halluzination ein.

Solche von Ärzten selbst erlebte und beschriebene Geisteskrankheiten dürften wohl zu den interessantesten Veröffentlichungen gehören. Dann gibt es noch manche von Ärzten im Wortlaut des Patienten veröffentlichte und analysierte Fälle, sowie einige von Nichtärzten veröffentlichte selbsterlebte Fälle. Von einigen älteren Datums möchte ich erwähnen: Kieser ${ }^{5}$ ), der einen Fall von Gehörshalluzination mit paranoidem Wahnsystem beschreibt, Engelke ${ }^{6}$ ), der einen Selbstbericht eines ,heiteren Wahnes“"veröffentlicht, Quince $\mathrm{y}^{7}$ ), der die Bekenntnisse eines Opiumessers wiedergibt. Der Fall bietet leider wenig Positives. Von neueren Arbeiten erwähne ich: Schreber ${ }^{8}$, Denkwürdigkeiten eines Nervenkranken. Auf 566 Seiten beschreibt der Verfasser seine eigene Paranoia als begutachteten Fall. Der Verfasser schreibt als Paranoiker seine Paranoia. Fehrlin ${ }^{9}$ ) beginnt unter dem Titel „Die Schizophrenie“ seinen eigenen Fall darzustellen. Im Laufe dieser Niederschrift mischen sich fremde Stimmen ein, die teils korrigieren, teils hemmen, allmählich aber als „Geister" die Beschreibung selbst in die Hand nehmen und das Ganze samt Analyse als Diktat enden lassen. James ${ }^{10}$ ) gibt die ekstatischen Erlebnisse in der ,religiösen Erfahrung" mit vielen Beispielen unter eigenem Wortlaut der Betreffenden wieder. Forel ${ }^{11}$ ) veröffentlicht einen Fall von Mania acuta, schildert seine Halluzinationen, Illusionen und Wahnideen. Gruhle ${ }^{2}$ ) gibt eine Selbstschilderung und Einfühlung, betont die Bedeutung der Selbstdarstellungen gebildeter Kranker. Jaspers ${ }^{12}$ ) gibt die Selbstdarstellung einer Schizophrenen im Alter von 32 Jahren (akute Psychose). Andere Selbstbeschreibungen finden sich noch bei Jas pers ${ }^{1}$ ) zitiert, ich erwähne hier nur die Autoren: Baudelaire, Ideler, Jakobi, Janet, Meinert, Nerval, Staudenmaier, Wollny, David.

Der vorliegende Fall gehört zu derjenigen Gruppe von seélischen Störungen, die sich der Beobachtung in der Klinik mehr oder weniger entziehen. An der Grenze des zur Anstaltsbedürftigkeit führenden Irreseins stehen eine ganze Reihe interessanter Psychosen, die für den Fachgelehrten verlorengehen, weil sie nicht in die Klinik kommen. Der Fall betrifft einen Herrn, der aus naheliegenden Rücksichten seine 
Erlebnisse nicht selbst veröffentlichen konnte. Die Halluzinationen stehen hier im Vordergrunde und müssen insofern Interesse erwecken, als sie Schilderungen eines Beobachters sind, der sich den Prozeß genügend $\mathrm{zu}$ objektivieren vermochte und dadurch die genauesten Erinnerungen an seine Erlebnisse behielt.

\section{Autobiographische Anamnese.}

Der Pat. ist 1878 geboren als zweites Kind gesunder Eltern. Bei Verwandten und Vorfahren sind keine Geisteskrankheiten bekannt. Mütterlicherseits ist in der Verwandtschaft Tuberkulose aufgetreten, die Mutter seltist frei davon (jetzt 76 Jahre alt). Der Vater ist in seinem 71. Jahre an kardialer Wassersucht gestorben. Fin Bruder ist gesund; sonst sind keine Geschwister vorhanden gewesen.

In der Kindheit traten bei dem Pat. keinerlei Krankheiten.auf; er litt viel an Pavor nocturnus, war von früher Jugend an trüumerisch, zurückgezogen, scheu, in der Schule stets verschlafen, er saB unterhalb der Mitte und hatte nie die nötige Aufmerksamkeit. In seinem 14. Lebensjahr verließ er die Realschule. Er hatte dort großes Interesse an Naturgeschichte, Zeichnen, Turnen und freier Betätigung gehabt. Sehr abgeneigt war er gegen Lernen aus Büchern (besonders Sprachen, Geschichte). Gegen Religion war er gleichgültig.

Seine moralischen Eigenschaften waren gut; er hatte stets Mitleid mit leidenden Tieren und Gerechtigkeitssinn, litt sehr bei häuslichem Zwist. Weinerlich veranlagt war er nicht. Er war sehr eigensinnig in bezug auf Erfüllen seiner Wünsche.

Schon in früher Jugend stellte er bei seinem Vater nicht zu beantwortende Fragen über das Warum aller Dinge, wollte den Naturereignissen auf den Grund gehen, war enttäuscht, ja betrübt, daß es dafür keine Antworten gab, wie er später sich wunderte, daB seine Lehrer vom Wesen des Lebens und die Theologen vom Tode nichts wußten.

Als Kind vom 3. bis 12. Jahre hatte er eine treue Kameradin aus der Nachbarschaft, schloß sich von anderen Kinderr ab. Mit Beginn der Pubertät verschwand das Interesse an ihr, obwohl von da ab die Beziehung zu anderen Mädchen nicht fehlte. Er verstand sich gut mit einem Freund von ähnlicher naturschwärmerischer Gesinnung, wie er sie selbst besaß. Die beiden lasen Bücher über Erfindungen und Entdeckungen. Als er den Freund mit dem 16. Jahr verlor, suchte er vergebens nach anderen Kameraden, die ihn verstanden; er liebte die Einsamkeit und das Nachdenken. Jugendstreiche machte er mit aus Nachahmung, ohne eigenes Interesse; er brach stets ab, wenn die anderen es gerade am schönsten fanden. Immer litt er unter einem undefinierbaren Suchen nach etwas, das er selbst nicht wußte. Schon mit dem 12. Jahr vertiefte er sich in die Werke von Jung Stilling.

Einen befriedigenden Beruf fand er nicht; er wollte sich zuerst dem Lehrfach widmen, kam aber davon ab und wurde Techniker.

Das körperliche Befinden begann vom 15. Jahr an ungünstig zu werden. Es traten Verdauungsstörungen auf mit chronischer Obstipation. Onanie wird nicht zugegeben. Der Zustand besserte sich mit dem 19. Jahr nach einer fortgesetzten physikalischen Behandlung. In den darauffolgenden zwei Militärjahren traten Erschöpfungszustände ein. Er bekam eine Cystitis gleich anfangs und war dann nach Heilung derselben 22 Wochen abwechselnd bei der Truppe und im Lazarett zur Beobachtung auf Herz und Lunge, da er während der Übungen über Atemnot klagte, bei Märschen zurückblieb usw. Fr hatte in dieser Zeit sehr unter dem $\mathrm{HaB}$ der Mannschaft zu leiden. 
Er wurde schließlich auf eigenen Wunsch wieder zur Truppe entlassen mit Schonung. Das zweite Dienstjahr verlief normal, er machte die Übungen außer Herbstmanöver und größeren Märschen mit, wurde im Herbst 1900 gesund entlassen und nahm seinen Beruf wieder auf.

Schon vom 18. Jahr an zeigte sich bei dem Pat. ein reges Interesse an Philosophic; Studien über Lebenskunst. Reformen. Er wurde abstinent in bezug auf Alkohol, lebte eine Zeitlang vegetarisch, verspïrte starkes Streben nach Vervollkommnung des inneren Lebens mit geringer Rücksicht auf das äußere Leben, er mied die Gesellschaft; das Lehen mit seinen Freuden erschien ihm nicht lebenswert. Er las eine Zeitlang naturwissenschaftliche Werke, dann philosophische (Kant, Schopenhauer, Darwin). Unbefriedigt daron suchte er in der spiritistischen Literatur (Davis, Du Prel, Aksakow) eine Lösung des Welt- und Menschenrätsels, ohne sich jedoch praktisch mit Spiritismus zu beschäftigen. Die Kirche bot für ihn nichts- Anziehendes.

Schließlich rang or sich zu einer vitalistischen Lebensauffassung durch und suchte den Aufblick zu einem Göttlichen, das er sich als alldurchdringendes BewuBtsein vorstellte.

Indem er immer mehr von diesen ldeen durchdrungen wurde. fühlte cr eine Besserung seines Gesundheitszustandes, ja er glaubte das Leben, indem er es quantitativ als Lebenskraft auffaßte, durch besondere Willens- und Gedankenkonzentrationen in sich vermehren zu können.

Diese Wendung bzw. praktische Wirkung anf seine Gesundheit verlegt er in sein zweites Militärjahr. Immer noch abstinent behielt er seine Gesundheit bis zum Eintritt in die Psychose.

Dieselbe begann 1/4 Jahr nach der Entlassung aus dem Militärdienst. Er hatte schon Monate vorher sehr auffallende, z. T. ihm schr angenehm erscheinende Gemütszustände herbeigeführt durch eine meditative Einstellung seines Bewußtseins.

Im Anschluß an cliese Periode traten plötzlich im Oktober 1901 Angstzustände, Verfolgungsgefühle, Gesichts- und Gehörshalluzinationen auf. Das Höhestadium wurde an Weihnachten 1901 erreicht.

Die Zustände gingen einher mit Schlaflosigkeit, Flucht in die Finsamkeit, großer Empfindlichkeit der Ninnesorgane, Verweigerung von Speisen. Anfang Dezember 1901 war der Hausarzt anwesend, der am Körper nichts Krankhaftes finden konnte. Fieber trat vor und während der Psychose nicht auf.

Der Urin war frei. Einc Infektion wurde nicht nachgewiesen. Kein Brbrechen, kein Schweißausbruch, keine Ohnmachten, kein Aussetzen des Pulsgefühls. Die Hautfarbe war hlaßgelb, der Ernährungszustand mäßig.

Vergrößerung der Schilddrüse lag njeht vor, die Sinnesorganlo waren intakt (der Augenhintergrund wurde nicht untersucht).

Von seiten der Lange und des Herzens keine Beschwerden. Pat. mußte so viel wie möglich den „Bauch“ leer halten, sonst kamen die „Dämonen“.

Keine Krämpfe. Mastdarm und Blase in Ordnung. Pat. gibt noch an, daß er zu manchen Zoiten sehr oft Stuhl und Urin entleerte, zu anderen gar nicht, daß er manche Tage fast alle 10 Minuten etwas essen mußte, zu anderen Zeiten tagelang nichts. Sprechen und Tnruhe in der Umgebung Iösten leicht die Verfolgungsideen aus. Ebenso starke Inanspruchnahme der Sinnesorgane. Pat. will durch Fasten und Erfindung von "Kraftworten. Schutzworten" (Gegenaffekte) die „Dänonen“ wieder überwunden haben. Die Rückkehr zu einem erträglichen Zustand kam nach etwa $1 / 2$ Jahr.

Die Psychose begann in Oktober 1901, hatte ihren Höhepunkt am 23. XII. 1901, blaßte dann sehr allmählich ab. Mit dem Wendepunkt bringt Pat. das Auftreten eines neuen Ichbewußtseins in Zusammenhang. Ganz befreit von der 
„Geisterwelt" will der Pat. auch heute noch njcht sein; er litt in den Jahren danach an kurzen Rückfällen nach heftigen Aufregungen oder körperlichen Anstrengungen. Heute (1918) macht der Pat. einen gesunden Eindruck.

\section{Selbstschilderung.}

„Indem ich meine Geschichte erzähle, bin ich mir bewußt, daß es nur ein Teil meines jetzigen lchs war, der dies alles erlebte. Bis zu dem 23. XIT. 1901 kann ich mich nicht mit dem Ioh von heute bezeichnev. Dies damalige Ich kommt mir jetzt vor wie ein kleiner Zwerg, der in mir sitzt. Fs ist für mein Gefühl unangenehm und für mein Existenzgefühl peinlich, die Erlebnisse bis dahin in der ersten Person zu schildern. Ich kann cs tun unter Anwendung von Gegenvorstellungen und indem ich mir bewußt bin, daß der "Zwerg' bis zu dem obigen 'Tag regierte, dann aber seine Rolle ausgespielt hatte.

Meine Kindheit war ohne besondere Freignisse. Ich hatte von Jugend auf Interesse an den Geheimnissen der Natur, wollte schon als Kind hinter den Schleier der Erscheinungen schauen, wandte mich sehr bald von den Freuden des Lebens $a b$, schon bald nacb der Schule lebte ich in einer eigenartigen Zurückgezogenheit; wenn ich bei Kameraden auch lustig erschien, so war dies nur obendrauf, alles Sinnen und Trachten ging mehr darauf aus, in reiner geistiger Anschauung zu leben; ich hob den Blick auf zu dem Ahsoluten, Namenlosen, Unbekannten, es bestanden keine persönlichen menschlichen Ideale, etwa Christus oder Heilige. Ich wollte die Tore aufstoßen, an denen .jeder gern vorüberschleicht"; wünschte mir die schlimmsten Krankheiten an, um zu sterben; selbst Hand an mich zu logen wagte ich nicht, da ich dies für einen mberechtigten Fingriff hielt.

In der Zeit der anhaltenden Interesselosigkeit gegen die äußere Welt (vom 17. bis 20. Jahr) tat sich cine innere Welt der Befriedigung auf. Nach dem 19. Jahr etwa entstanden während dieser. Konzentrationen der Gedanken auf das Absolute gewisse Vibrationen, die den ganzen Körper durchzogen, ein Gefühl der Wonne, des Ausgeglichenseins, des Friedens, das sich immer stärker entwickelte. Ich war unwillkürlich Anhänger eincr vitalistischen Weltauffassung geworden, ich moßte eine den Raum ausfüllende Lebenskraft annchmen. Gelesen hatte ich einiges darüber. Bei geschlossenen Augen wurde in diesen Zuständen diffuses milehweißes Licht wahrgenommen, aus welchem oft in leuchtenden Farben wunderbare exotische Pflanzen- und Tierformen sich abhoben. Das Dämmerlicht schien mir in den Augen selbst zu liegen, aber die Formen waren wie ein Seelenerlebnis, tauchten aus einer anderen Welt auf.

Die Wahrnehmung des Lichtes war nicht immer gleich. Wenn meine Seelenverfassung gut war, war es heller, aber nach geringem moralischem Minus ( $z$. B. nach Ärger, Erregung) oder nach körperlichen Disharmonien (z. B. nach zuviel Essen) war es dunkler oder es trat völlig rabenschwarze Nacht ein. Wenu es dunkel war, wußte ich auch immer, daß wieder etwas nicht in Ordnung war. Dies Licht trat nach etwa 1-2 Minuten ein, wenn ich die Augen schloß. Es war nicht Licht, das von außen hereindrang. Wenn ich mit der Bahn durch einen Tunnel fuhr, die Angen schließend, dann wurde es bald hell; ich meinte dann irrtümlicherweise, der Zug sei bereits wieder in Freien. Als ich dann plötzlich die Augen öffnete, umfing mich noch die absolute Nacht des Tunnels. Das Licht verschwand nicht gerade deshalb, weil ich die Augen öffnete, sondern weil ich mich bemühte, mit dem Auge nach außen zu sehen. Sobald ich nicht mehr fixierte, konnte ich auch bei geöffneten Augen, sogar bei Tag, dieses Licht sehen, nur undeutlich.

Die Formen traten nicht jederzeit auf, sondern etwa alle 2-3 Tage einmal. Die Pflanzen waren nicht so, wie ich sie mir in der Vorstellung hätte schaffen können, ieh erstaunte ïber die Schönheit und Grazic der Form, es lag etwas Prunk. 
haftes darin, es war, als seien die mir bekannten Pflanzenformen nur degenerierte Abkömmlinge davon. Die Tierformen waren vorsintflutlichen Arten ähnlich, hatten alle etwas Gutartiges. Nanchmal fielen extrem hervortretende Teile auf, aber ich wunderte mich, wie harmonisch doch die ganze übrige Körperform an jene Einseitigkeiten angepaßt war, so daß ein Typus dadurch zum Ausdruck kam. Seltıst die groteskesten Formen machten einen natürlichen lebensfähigen Eindruck. Bewegung hatten sie nicht, sie traten als plastische Formen auf und verschwanden nach einigen Minuten wieder. Das Schauspiel machte auf mich Eindruck, füllte das ganze Bewußtsein aus, verursachte ein gewisses Hingegebensein im Empfinden. Die Formen veränderten, bewegten sich nicht.

Die Empfindung einer durch den Körper strömenden Lebenskraft wurde immer stärker, der Körper wurde gesünder und kräftiger, eine seit Jahren bestehende Verdauungsträgheit verschwand vollständig, nachdem andere Anwendungen nur wenig genützt hatten. Diese Wirkung machte sich während meines zweiten Militärjahres geltend (im 21. Jahr); ich lernte durch diese Übungen gewisse körperliche Schwächezustände überwinden.

Dies Leben in einem inneren Reich sollte nun doch nicht ungestört bleiben, es mischten sich (etwa Oktober 1901) Dinge sehr unangenehmer Art ein. Schon im Sommer 1901 wurde mein Bewußtsein durch diese Meditationen sehr herab. gedämmert.

Ich hatte die Meinung, auf diesem Wege endlich in eine übersinnliche Welt vordringen zu können, und hatte das Gefühl, als sei nur noch ein dünner Sohleier zwischen mir und dieser jenseitigen Welt. Ich fühlte mich oft wie aufgeteilt in die Dinge der Umwelt.

Eben hatte ich wieder einen weiteren Schritt in diesem Selbstvergessen ge. tan, als sich drohende Elemente erhoben, die nicht nur jedes weitere Eindringen in diese innere Welt, dje für mich Durchgangspforte zur übersinnlichen Welt sein sollte, verhinderten, sondern im Laufe der nächsten Monate zu zeigen schienen, daß ich mich ungeahnt schon längst in die Hände dieser Elemente ganz gegeben, es nur nicht béachtet hatte.

Im Oktober 1901 traten Angstzustände und schreckhafte Träume auf, deren Herkunft mir nicht klar war. Die Nächte wurden immer unruhiger, und drohende Gestalten sowie Stim men traten auf. Die Gestalten gruppierten sich um mich herum in einem Abstand von 3-6 m. Es waren groteske Menschengestalten, die anfangs nicht deutlich zu erkennen waren, anscheinend auch weiter entfernt waren und einen Lärm verursachten, wie ein Stimmengewirr von sich um nichts streitenden Krämern und Händlern, wobei jeđer den anderen in höchstem Eifer und in häßlichster Weise übertönen will, jeder in wahnhafter Weise nur das hört, was er selbst sagt; es war ein Geräusch, als seien etwa einige Hundert solcher in einem für sie zu kleinen Saale zusammengepfropft, sämtliche johlend und schimpfend, ab und zu einzelne kräftigere Stimmen hervortönen lassend.

Die Gestalten waren im Raum, aber es war, als hätten sie ihren eigenen, ihrer Wesensart zugehörigen Raum. Dieser neue Raum mit seinen Bewohnern trat um so deutlicher auf, je mehr meine Sinne von den bekannten Dingen abgelenkt waren. Tch konnte die Fntfernung genau angeben, aber die Gestalten waren nie von Gegenständen des Zimmers abhängig, wurden nie durch solche verdeckt. Sie konnten nie zugleich mit einer Wand, einem Fenster u. dgl. wahrgenommen werden. Auch später, als ich die Gestalten willkürlich betrachten konnte, zeigten sie sich mir nur. wenn ich mir die übrigen sinnlichen Dinge hinwegsuggerierte.

Diese Gestalten wechselten zum Unterschied gegen die früheren manchmal ihre Form; ich sah harmlosere Formen sich in drohendè umwandeln, oder später, als ich sie beeinflussen konnte, sah ich sie einschrumpfen und zusammensinken. 
Die Farke war meist grau, manchmal schien aber eine hervorstechende Farbe etwas Besonderes andeuten zu sollen, z. B. bei gelben Augen, hochroten Lippen, schwarzer Hautfarbe usw. Die Gestalten schienen eine übertriebene Personifikation kleiner geringster Fehler zu sein, die ich selbst machte (ohne daß ich mir darüber Selbstvorwürfe gemacht hatte), ganz harmloser Unaufmerksamkeiten in hygienischer, ethischer, moralischer Beziehung, ja sogar oft eine unverschämte Verzerrung rein physiologischer Vorgänge; z. B. wenn ich bei Tisch den Geschmack einer Speise angenehm empfand, so konnte am selben Abend (als die Speise sohon verdaut war) als Echo dieser Empfindung ein Dämon sich melden in der Gestalt eines gefräßigen, lüsternen Menschentieres, mit großem Maul, wollüstigen dicken roten Lippen, dickem Bauch, riesenhafter Größe. Ich fühlte dann nachher noch so lange seine Nähe, bis ich mich eine Zeitlang (etwa 2-3 Mahlzeiten) des Wohl. geschmacks (wie als seiner Nährquelle) enthalten hatte. Auch wenn ich sehr müde mich hinsetzte und mich so recht dem Gefühl des Ausruhens hingab, entstand ein diesem Gefühl entsprechender Dämon. Auch eine bloße Gegenrede, wenn Vater oder Mutter etwas kritisierten, erzeugte eine ungeheuere bösartige Gestalt, die das Ich zu erdrücken drohte. Nach Kränkung sah ich düstere, träge Gestalten, die den Eindruck des Ungesunden machten.

Die Einwände meiner Umgebung, diese Dinge würde ich mir nur vorstellen, konnte ich nicht akzeptieren; ich konnte zwischẹn Vorstellungen und meinen Wahrnehmungen njchts Verwandtes finden, auch heute nicht. Bei Vorstellungen kommt es mir vor, als seien sie in gar keinem Raum, blieben als matte Bilder in meinem Gehirn oder hinter den Augen, während ich bei meinen Wahrnehmungen von außen her eine Welt erlebte, dic aber doch auch die Sinnenwelt nichts anging. Alles, was sie enthielt, war für mich wie die Wipklichkeit, die Formen voller Leben. Auch später noch war für mich in der gewöhnlichen Welt noch eine andere mit ihrem besonderen Raum, und das Bewußtsein glitt nach Belieben hinüber oder herüber. Beide Welten waren gleich lebensvoll. Vorstellungen kann ich ebensowenig mit den Wahrnehmungen der einen als mit denen der anderen vergleichen.

Etwa im November 1901 wurden die Gestalten deutlicher, zeigten aber so einseitige unsymmetrische Körperformen, eine solche Existenzwidrigkeit, daß Entsetzen und Abscheu in mir erweckt wurde. Z. B. es erschienen Gestalten, bei denen ein Organ auf Kosten der anderen enorm groß ausgebildet war. Ab und zu rief eine deutlichere Stimme mir zú (und in diesem Moment war der ganze übrige Lärm still): ,Der gehört auch zu uns, der ist auch verloren wie wir, er hat umsonst gelebt." ,Der kommt zu uns, bald wird er bei uns sein." Die Stimmen waren wie durch Echo verstärkt oder wurden oft plötzlich stärker oder schienen wie in Form von Geschossen heranzukommen und ganz in der Nähe einzuschlagen. Sie kamen aus der Ferne, schlugen aber in das Ohr ein, wie wenn jemand einem durch ein Sprachrohr ins Ohr spricht.

Diese Zustände gingen nachts oft fort bis frühmorgens 4 oder $6 \mathrm{Uhr}$, in dieser oder einer anderen Form. Diese Dinge waren für mich so real, daß ich mir im Moment nicht klarmachen konnte, daß es keine Wirklichkeit sei. Ich mußte oft aus dem Bett heraus und eine Viertelstunde im Zimmer hin und her gehen, um mich wieder auf den festen Boden des Daseins zu bringen. Beim Nachlassen ging es aber wieder von neuem los.

Allmählich fühlte ich mich noch näher dieser Gesellschaft von Dämonen. Der Lärm verschwand, aber nun stürzten sich oft einzelne grauenerregende Gestalten auf mich zu und schrien mir etwas ins Ohr. Es war, als seien sie aus einem Versteck von hinten oder von der Seite herbeigekommen, und zwar in Lebensgröße, früher waren sie der Entfernung gemäß kleiner. Ich hatte nun das Gefühl, als sei ich ständig unter Verbrechern und Teufeln, die ich sah und hörte, sobald die gespannte Aufmerksamkeit auf die sinnlich wahrnehmbaren Gegenstände der 
Umwelt nur um das Geringste nachließ. Es war jedoch nicht immer die Kraft vorhanden, die Aufmerksamkeit von diesen Dämonen abzulenken resp. sie auf die sinnlich greifbaren Gegenstände zu lenken. Jeder Versuch, dies zu tun, glich der Bemühung, einen Mühlstein auf einen Berg zu wälzen. Z. B. des: Versuch, ein Gespräch eines Bekannten anzuhören, das mehr als einige sätze enthielt, war von einer derartigen Unruhe gefolgt (weil drohende Gestalten sich auftürmten), daß rasche Entfernung resp. Verabschiedung nötig wurde. Dasselbe geschah beim Versuch, einen Brief, eine Karte zu schreiben; dies ging nur in abgebrochenen Sätzen, Einzelworten, ich war dabei von drohenden Gestalten umringt, die nicht zuließen, daß ich schreibe. Ich erinnere mich z. B. cine Karte geschrieben zu haben, die immer nur die ersten und letzten zwei Worte eines Satzes enthielt, z. T. noch weniger, dás übrige füllte ich durch Punkte aus. Ganz schwierig war es, die Anfmerksamkeit einige Zeit anf einen Gegenstand zu richten; mein Geist glitt sofort ïber in andere Regionen, aus welchen dann hald, wie herausgefordert, die Dämonen auf mich losstürzten.' Ich nuß hervorheben, daß dieses Übergleiten oder Nachgeben anfangs freiwillig geschah, von mir gesucht wurfe. wobei jener angenehme, harmonische, von der Welt abgeschlossene innerliche Zustand eintrat. damals ohne Dämonen, wie jch weiter oben beschrieh; jetzt ging aber dieses Übergleiten wie von selbst, ja es war eine Schwäche. ich fühlte mich unwiderstehlich dazu getrieben. Der Übergang vom gewöhnlichen Zustand in die Welt der Dämonen geschah jetzt ganz unmerklich, so daß ich erst hinterher oft merkte. was geschehen war. Am meisten kam dies vor bei einer Veränderung des körperlichen Zustandes; jede Schwankung des Stoffweehsels war gleichsam eine Einlaßpforte. Auch Sinneseindrücke, z. B. eine rasche Einstellung des Auges auf Kintfernung, grelles Licht oder das Hören angenehmer Töne gaben Anlaß. Hinterher wurde erst durch die Folgen erkannt, was geschehen war. Etwa $1 / 2$ Stunde danach kam wie als Reaktion das ganze Heer der Dämonen. Waren sie cinmal da. so wirkten allerlei Dinge für sic begünstigend. Die Magen- und Darmfunktion spielte auch eine große Rolle bei diesen Bedrängnissen. Bei gefülltem Magen waren sie stärker. Wenn Speisen lange im Darm verweilten. waren die Dämonen schwer ahzuhalten, die Bewußtseinsherabdämmerung war für mich dann noch viel folgenschwerer. Abends, beim Versuch zu schlafen, schon beim Augenschließen war der Eintritt in den Wirbel unvermeidlich, während ich ihn bei Tag eher hintanhalten konnte; es war ein Gefühl von Im-Kreise-gedreht-Werden, wonach dann bald die Gestalten auftraten. So mußte ich stets schart wachend und aufpassend im Bett liegen, bis nach Stunden der Feind sich etwas verzog. Das einzige. was ich tun konnte, war, die Sache nicht freiwillig durch ,Sichgehenlassen- zu befördern; geschah dies doch, dann waren die Folgen fürchterlich; es kamen Rufe der Verhöhnung, Vorwürfe usw. Das Kommen und Gehen der Dämonen ging nach einem System, das ich später genau durchschaute (weiter unten beschrieben); damals wußte ich nur: es lief programmäßig ab wie ein Theaterstück. Etwa 1/2 Stunde nach der Bewußtseinstrübung (die nur 1 Minute zu dauern brauchte) war es, als ginge in mir ein Vorhang auf und die einzelnen Etappen des Dämonenanfmarsches folgten aufeinander; es dauerte etwa $1 / 4$ bis $11 / 2$ Tage. Es gab dabei Stunden, in denen ich gar nichts erlebte, ich wubte aber stets, welche Stelle des Programmes gerade im Umlauf war. An Schluß hatte ich den eigenartigen Eindruck, als stehlen sich dic Beteiligten einer nach dem andern wieder fort, und ich fühlte mich von einem bodenlosen Abgrund oder ich möchte sagen Meere des Wahnes wieder auf festes rand gesetzt. Ich vergleiche hier nicht, sondern meine es wörtlich; ich hatte das leibliche Gefühl eines Menschen. der nach einer Seefahrt wieder auf Land kommt.

Wie ich später erkannte, gab es zweierlei Qnellen für meine Bedrängnisse: J. Als Hauptursache die eigenartigen süßlichẹn Gefühlsimpulse, entweder 
freiwillig gesucht oder entlockt und kegünstigt durch Sensationen. Der Kreislauf der Erlebnisse wurde dadurch in Gang gesetzt.

2. Die verstärkenden Trsachen, z. B. Affekte und körperliche Unregelıäßig. keiten, die die vorhandene Situation verschlimmerten, z. T. den Dämonen ihren Typ gaber.

Punkt 2 war mir immer klar, der Typ der Gestalten zeigte mir ja stets selbst in belehrender Weise. was jeweils ihre Nährquelle war. Was Punkt 1 betrifft, so ahnte ich damals nicht den Zusammenhang mit der Sache, hätte mich auch von niemandem darüber aufklären lassen, hätte auch gar nicht die Macht gehabt, dagegen anzukämpfen; im Gegentei], ich hätte denken mü̈ssen, ich kämpfe in der mir nachteiligen Richtung; ich war so eingewoben von einer gewissen Zeit an, daB ich fast behaupten möchte, diese Gefühlsmomente gehörten selbst zu meinem Wahn.

So gab es denn zunächst keinen Ausweg aus dem Labyrinth. Ich konnte schon längst nichts mehr arbeiten, lag nur auf dem Bett oder saß im Lehnstuh], hielt jede Unterhaltung mit Menschen ängstlich ab. Hilfe von anderen Menschen war unmöglich, ein Trost konnte die Sache selbst gar nicht berühren, aufmunternde Reden brachten Unruhe. Fin geringes Milfallen von seiten der Umgebung hetzte eine Heerschar von Dämonen auf mich. Bei Gegenwart des Arztes trat Unruhe ein, I'mherlaufen, das Gefühl, als sollte ich durch irgendeine Öffnung entrinnen. Am besten wirkten Menschen mit ganz passivem Verhalten, z. B. das schweigende Verhalten eines Freundes, Bruders, Vaters. Geräusche von unbelebten Dingen störten nicht.

Gegen Mitte Dezember verschlimmerte sich der Zustand so sehr, daß Vorfolgungsideen auftauchten: ich flüchtete mich vor meiner Mutter, suchte mir aus Verzweiflung die Haare auszuraufen. Dann fühlte ich mich wieder wie ein Kind. Das Jchgefühl war so kJein, daß das Bedürfnis auftrat, es durch eine andere Person zu ergänzen, sowie das Verlangen nach der schützenden Nähe stärkerer Iche, ruhiger, sich selbst beherrschender Menschen, die die Dämonen abhalten sollten. In frühere Zeiten fühlte ich mich nie versetzt, auch lebte ich nicht in meiner eigenen Kindheit, sondern ich kam mir vor wie nur ein Bruchstück eines Menschen. Dies Gefühl war nicht seelisch, sondern seelisch-körperlich, so daß ich, hätten meine dugen mich nicht eines anderen belehrt, wirklich hätte glauben müssen, ich bestehe nur aus einer Hälfte oder ${ }^{2} / 3$ eines Menschen.

Es traten diesem verkleinerten und zerschmetterten Ichgefühl entsprechende 'Träume auf, meist im halbwachen Zustand. Z. B. sah ich ein Kind mit einer Schnur an eine Kirchturmspitze gebunden; die Schnur riß, das Kind fiel herab und war zerschmettèrt. Beim Erwachen fühlte ich mich selbst als dieses Kind; in meiner Seele war dann eine unendliche Zerrissenheit, oder richtiger: Krüppelhaftigkeit; erst nach einer halben Stunde etwa ließ diese Wirkung etwas nach.

Die Nächte waren schlaflos bis morgens etwa 6 oder 7 Uhr unter fortwährendem Kampfe. Allmählich wurde mir klar. daß ich mehr Ruhe hatte, wenn ich abends nichts Festes mehr zu mir nahm. Diese Einschränkung mußte bald noch verschärft werden, so daß ich nur mittags noch feste Nahrung aufnahm, um dann nachts 4. Uhr oder später endlich einschlafen zu können. Aber dennoch nahm der Prozeß seinen absteigenden Verlauf. Riesenhafte Ungeheuer schọssen jetzt mit großer Geschwindigkeit auf mich ein, ich fühlte mich in diesem. Moment wie papierdünn zusammengepreßt, fühlte mich in großer Gefahr, das Ich zu verlieren, und merkte deutlich, daß dies Wahnsinn oder Blödheit bedeutete. Nach einer halben Minute ging ein solcher Dämon wieder fort, nachdem der heftigste Kampf stattgefunden hatte; doch bald kehrte or in anderer Form wieder. Danach kam es mir oft vor, als sehe ich dem Dämon ähnlich; dies war jedoch nur ein Gefühl; Grimassen habe ich nicht geschnitten.

Meine Art der Verteidigung wurde oft gewechselt. War ein Dämon da, dann gab es kein Mittel, als nur das Ich festzuhalten, damit es nicht verlorengehe. 
Im übrigen machte ich alle möglichen Versuche. Ich mußte mich wie einem bösen Tier gegenüber verhalten, bei dem man nie weiß, durch welche Kleinigkeiten etwa man seine $W u t$ und Raserei anfachen wird. Einmal muBte ich stillsitzen, um den Feind nicht zu wecken, ein andermal umherlaufen, um ihn nicht zu ärgern. Einige Tage hindurch mußte ich jede Viertelstunde etwas essen, sonst nahmen die Bestien die drohendste Miene an; ein andermal durfte ich einen ganzen Tag nichts essen, um sie nicht zu reizen. Alles half im Grunde nichts, ich blieb in ihrem Bann.

- Wenn ich imstande war, die Feinde einige Zeit direkt abzuhalten, so suchten sie andere Wege, um mein Bewußtsein wieder von der Wachsamkeit abzulenken, benützten angenehme Sinnesreize als Eingangspforte, schöne Farben, das Betrachten von Gemälden, das Auffallen hellen Lichtes auf die Augen, angenehme Töne, Musik oder das bloße Sprechen von Leuten.

Ganz besonders auf dem Wege der Nahrungsaufnahme gelang es dem Feinde hereinzukommen. Ich fühlte mich dann, solange überhaupt etwas im Darm war, wie eingefangen.

Andererseits wurden Affekte für mich immer bedenklicher, besonders Zorn, Ärger, Haß, Mißtrauen. Auch die der anderen Menschen; ich sah bei allen Menschen der Umgebung die kleinsten Charakterfehler als häßliche oder drohende Gestalten, die aus ihnen heraustraten und auf mich losstürzten; ich wünschte sehr einen Menschen zu finden, der keine Fehler hat, oder der seine Dämonen beherrscht, denn ich glaubte, der hätte auch die meinigen beherrscht.

Ich war mir meiner Lage bewußt und beschloß nun alles zu meiden, was dieselbe verschlimmern konnte; aber damit war ich ja an einem Ende angelangt; denn ich wußte, dies bedeutete nach Obigem nicht mehr essen, nicht mehr trinken, nicht sehen, nicht hören, nicht genießen, mit niemandem mehr verkehren; aber dies hieß sterben. Ich war entschlossen zu sterben, um wenigstens das Ich zu retten. Die Dämonen wollten, der Körper solle leben und das Tch solle sterben, sie waren jederzeit bereit, zu meinem Nachteil den Körper kräftig zu machen. Ich aber wollte, das Ich solle leben und der Körpor sterben, und ich war bereit, letzterem alles zu entziehen. Während der nun folgenden vier Dursttage und sechs Hungertage kam es jedoch zu einem Kompromiß und es trat eine ganz andere Lösung der Frage ein.

Ich hatte bis dahin allmählich bestimmte Einsichten gewonnen. Vorher hatte ich jenes angenehme Schwindelgefühl (Punkt 1), das nur kurz anhielt, aber sozusagen alle Seligkeiten in sich schloß, immer noch nicht als in Zusammenhang stehend mit den Dämonen, die dann tagelang auftraten, erkennen können. Erst später, als ich mich schon dauernd in der Gesellschaft oder Nähe oder im selben Raume mit den Mißgestalten fühlte, als ich dann sehr wenig aß, kam mir diese Frkenntnis ganz allmählich in Form eines inneren Schauens im völlig wachen Zustand. Das ganze Kommen und Verschwinden oder Abflauen der Gestalten wurde nun noch genauer als ein Kreislauf erkannt. Ich glaube, daß nur durch das Fasten jetzt mein Blick so geklärt wurde, daß ich den Zusammenhang klar durchschauen konnte. Mit einer besonderen Objektivität konnte ich jetzt alles verfolgen.

Wenn nach stundenlangem Kämpfen und mühevoller Beherrschung aller Sinne unter allmählichem Abflauen der Gestalten das seelische Gesichtsfeld frei und leer geworden war und das Bewußtsein wieder mehr der äußeren Welt zu. gekehrt war, trat ein gewisses Heimwehgefühl auf; diese irdische Welt kam mir vor wie eine große Illusion, ich fühlte mich auf der Erde wie im Exil, es kam mir oft vor, als seien alle seelischen früher vorhandenen Verbindungsfäden zwischen mir und dem Wesen der Dinge zerrissen. Im Anschluß daran fühlte ich mich in der Mitte eines dämmerhaft erhellten Raumes (besonders abends wurde mir diese Situation sehr deutlich). Dann trat in überredender oder 
verführerischer Weise eine Gestalt auf, die etwas geistig Überlegenes und Angenehmes in ihrem Auftreten hatte. Mit einer vortrefflichen Überredungs. kunst unter strenger Logik, teils in Worten, ganz kurzen Sätzen, teils in Vorstellungen, die wie in meinem Gehirn ablaufend erfaßt wurden, als ob das Gehirn dies wie eine Uhr mechanisch zum Ablauf brächte, teils auch in Bildern, wurde niir nahegelegt, daß ich wieder glücklich werde und an dem Glück aller anderen teilhaben werde, mit allen Wesen der Welt glücklich verbunden sein werde, wenn ich nur eine einzige Bedingung erfülle; in diesem Moment war es, als würde mir von irgend jemandem eine Art Hostie dargereicht, öfters auch ein Gefäß mit einem Getränk, und ich fühlte mich zu dem früheren seligen Dahinschwindenlassen des Bewußtseins eingeladen. Dies sollte mich also retten. Ich ging darauf ein und nach 10-30 Minuten verwandelte sich die Szene vollständig. Ich fühlte mich wie in einer Spirale gedreht, es lief ganz programmmäßig wieder das Folgende ab: Es entstand der frïher beschriebene Lärm, wie wenn viele Personen miteinander streiten, danach kamen die grotesken Gestalten, dann die drohenden, verhöhnenden, lästernden Zurufe, die riesenhaften Ungeheuer, die über mich herfielen, die die grauenhafteste Seelenangst erzeugten mit dem Gefühl, vernichtet zu werden, dann die Bedrängnis des Ichgefühls, das wie eine ganz klein geschraubte Flamme wurde, das am Erlöschen zu ewigem Blödsinn ist.

Nachdem nach 6-24 Stunden die Bühne wieder leer war, was nur nach größter Anstrengung zustande kam, begann derselbe Kreislauf von neuem. Die Dauer und Schwere des Anfalls richtete sich immer nach der Intensität, mit welcher ich von Obigem ,trank" oder , aß*. Mehrmals unterlag ich noch dieser Versuchung, nachdem ich sie schon als solche erkannt hatte. Denn im Moment wirkte diese wie ein Muß, wie eine Suggestion. Ich war nicht in der Lage zu opponieren, denn in diesem Augenblick konnte ich nicht erfassen, da $B$ ein solches verheißungsvolles Gefühl von Beglückung eine Hölle zur Folge haben solle, wenn ich es auch hinterher einsah.

Im Laufe dieser sechs Fasttage erwarb ich mir aber volle Klarheit. Es war, als stünde ich mehr über der Sache und der ganze Ablauf kam mir so gemacht vor. Als die überredende Gestalt auftrat, nahm ich einen mehr kritischen oder objektiven Standpunkt ein. Als die betäubende Substanz gereicht wurde, bekam ich Mißtrauen, ich zwang mich unter Aufwand aller Kräfte zu einem inneren Nein. Dies w̧ar etwa am fünften Fasttag. Die Folge davon war, daß der Logiker resp. Versucher sich in eine häßliche Gestalt verwandelte, als hätte ich ihn gezwungen, sein wahres Gesicht zu zeigen, um dann zusammenzuschrumpfen. Zugleich hatte ich ein fast unerträgliches körperlich-seelisches Leidensgefüh]. Ich hatte die Empfindung, als würde mein ganzer Körper in Stïcke zerrissen, alle Organe herausgerissen. Danach fühlte ich mich aber frisch. Nun trat aber diese Versuchung noch in anderen Formen wieder auf. Es kam wieder ein Gefühl der Verarmung und entstand ein eigenartiger seelischer Durst. Nach einiger Zeit des Schmachtens trat eine schöne, in Farben strahlende Gestalt auf mit etwa einem Kelch oder Becher und stummem Hinweis, als sei hier meine Rettung. Das Dargebotene sah ich nicht gleich, sondern erst hinterher, nachdem ich in dem Anblick der Gestalt fast wie berückt war; ich weiß auch nicht, ob die Gestalt selbst den Becher hatte, ich glaube eher, es.war eine andere Hand, die ihn irgendwo aus einem Versteck herreichte. Sofort kam das Mißtrauen, ich sagte wieder ein entechiedenes Nein und die Szene verschwand mit samt dem Durst. Dies Verschwinden ging sehr rasch; es war, als hätte ich durch ein Zauberwort alles vernichtet.

Nun sah ich mich wie in einer Einöde, ich fühlte Hunger und Schwäche; da waren allerlei Geschöpfe in halkiverhungertem Zustande. Es überkam mich ein großes Mitleid und das Gefühl, daß ich an diesem Elend schuld sei, denn ich 
glaubte, daß durch mein Vorgehen ihr jetziger Zustand entstanden war. Sobald ich dies Mitleid empfand, tauchte eine Gestalt auf wie die eines barmherzigen Samariters und sagte etwa: An dir allełn liegt es, diese da und die ganze übrige leidende Welt zu erretten, sieh diese Elenden, nimm dies! In diesem Moment sah ich, daß ich ein falsches Mitleid hatte, es war der Art, wie man es mit sich se]bst und seinen liebgewordenen Gewohnheiten hätte. Jetzt wußte ich, daß diese Elenden meine Dämonen waren. - .. Nach abermaligem entschiedenem Nein, das mir erschütternd durch alle Knochen schoß, und in der festen Absicht, lieber selbst zu sterben, verschwand plïtzlich auch diese Szene mitsant dem Hungergefühl, der Schwäche und deın Mitleid. Ich fühlte mich dann merkwürdigerweise sehr gekräftigt, es war mir, als wäre alle Lebenskraft dieser sterbenden Dämonen nun in mich übergegangen.

Dann trat aber wieder eine trostlose Öde und Verlassenheit in mir auf, die ganze Welt kam mir wie erstorben vor, ich hatte ein Gefühl, als wäre die Auflösung des Weltsystems nahe, und hatte auch diesbezügliche Visionen. In diesem Augenblick wußte ich, daß die sterbenden Dämonen Teile meines Tch gewesen waren, das jetzt wie zerbröckelt dalag.

Unter diesen Eindrücken prägte jch den Gedañken. selbst in alle Ewigkeit nichts mehr von der berauschenden Substanz nehmen zu wollen. Behaftet mit diesem Gefühl der Vereinsanung und einem Ich, das nur ein kleiner Bruchteil eines Ganzen war, verbrachte ich in Ausdauer noch etwa 36 stunden. Dann trat neben dem bisherigen Ichgefühl ganz plötzlich und unvermittelt ein zweites, viel stärkeres Ich auf, das bisher nie gefühlt wurde, mir nie bekannt war. Tch hatte das Gefühl. als hätte dieses Ich seinen Sitz etwa in der Magengegend oder hinter der Magengegend und würde sich in ganzen Körper strahlenartig ausbreiten.

Dies war am Finde des sechsten Fasttages, etwa am 23. Dez. 1901. Nun kehrte ich wieder zum Leben zurück. Ich fand in diesem Ich von nun an den höchsten und einzigen Schutz gegen alle künftigen dämonischen Anfechtungen. Ich fühlte es als das wirkliche Ich, das andere friihere als ein weniger wichtiges. ja als ein ganz unwesentliches Ich.

Dieses Verhältnis ist bis heute geblieben.

Inı Denken dieses neuen wirklicheren Ich wurde der Körper wie ein Rock gefühlt. den man an sich trägt, d. h. dieses Jch konnte nicht mit dem Körper identifiziert werden: es war jederzeit die Zuflucht zu diesem überpersönlichen. starken Jch möglich und geschah, wenn nötig. durch innerlich ausgesprochene Erinnerungsworte. symbolische Handlungen u. dgl.

Mein körperliches Befinden hob sich von diesem Tage an sofort. Bei jeder Sinnesempfindung hatte ich die Gewißheit, daß nur ich daran beteiligt war und keine fremden feindlichen Dämonen. Nach dem sechsten Fasttage konnte ich auch direkt Nahrung aufnehmen, ich hatte das Gefühl, daß nur ich selbst jetzt esse. trinke, sehe, höre usw., während ich früher stets die Empfindung hatte, als ob Fremde dabei mitwirkten oder durch diese Vermittlung eines Sinnenvorganges sich einschleichen wollten. Ich fühlte mich jetzt zwar auch noch umlagert und sah und hörte jene Gestalten von früher (mit meinem Leben lebte auch das ihrige wieder auf), aber sie konnten nicht mehr das Ich verdrängen; sie mußten sich in gewissem Abstand halten. Sic lagen beständig auf der Lauer und suchten jede Gelegenheit zu erhaschen, nahe zu kommen. Solche Gelegenheiten waren Gemütsbewegungen und Affekte aller Art, körperliche Anstrengungen, Ungenauigkeiten im Essen und Trinken. Am schlimmsten wirkțen Zorn und Ärger und körperliche Strapazen. Günstig dagegen wirkte geistige Arbeit.

In den ersten Monaten glich mein Ailgemeinzustand noch dem eines Schwerkranken, der eben von einer großen Operation auferstanden war und bei dem die größte Gefahr einer Nachblutung und Verblutung vorliegt. Ich ging vorsichtig 
auf der Straße umher wie einer, der ein Glas mit Wasser zu tragen hatte, das bis zum Rande zum Überlaufen voll war und jeder Verlust eines Tropfens ein Todesurteil bedeutete. Dies hatte ich nicht als Bild, sondern im Gefühl, ich führe es nur zur Illustration an. Das Ich war noch so labil, noch so wenig gefestigt, daß ich noch Sorge haben mußte, es wieder zal verlieren. Als Bild hatte ich lange Zeit folgendes: Ich sah das neue Tch als neugeborenes Kind, das sorgsam zu hüten war; von ihm ging alles Leben und alle Kraft aus, aber es konnte meinen Körper noch nicht ganz durchdringen, er war zu groß, ich hatte oft das merkwürdige Verlangen, man möge mir ein Bein oder einen Arm abnehmen, damit der Körper ganz ausgefüllt werde. Später wurde dies bess $\odot$, ich fählte sogar zuletzt das Ich über den Körper hinausragen, in den Raum hínein.

So gab es anfangs Rückfälle, wodurch die Gestalten wieder auf kurze Zeit äber mich herfielen. War das Gleichgewicht wiederhergestellt, dann waren die Gestalten eingeschrumpft abgemagert, spindeldürr, ohnmächtig. Ich sah immer, wenn ich wollte. diese Gestalten und konnte daraus auf meinen eigenen Zustand schließen. Jede geringste Störung kündigte sich durch das Aussehen dieser Gestalten an. Jede Disharmonie des Körpers, irgendeine Erkältung, ein Schnupfen, kündigte sich schon vor Ausbruch durch Gestalten an: sie hatten belehrenden ('harakter.

Um die Herrschaft über diese Ding " zu behalten, fühlte ich nich gezwungen, innere Schutzworte auszusprechen; rie hatten den Zweck, mich auf das neue Ich, das sich ab und zu verschleiern wollte, kräftiger zu besinnen. Z. B.:

,Ich bin' (dabei suchte ich das neue, nicht das alte Ich zu fühlen).

"Ich bin das Absolute" (jch meinte dies dem Leiblichen, Gewordenen gegenüber, ich wollte nicht selbst Gott sein).

,Ich bin der Geist, nicht der Leib.

.Ich bin das Fine in Allem.

.Ich bin das Dauernde' (den Schwankungen meines Jeiblichen und seelisehen Lebens gegenüber) oder ich wandte nur einzelne Worte an, wie: , Kraf1 , , Leben".

Am besten wirkten kurze Sätze, die das Ich als etwas den Körper Durchdringendes, aber vom Körper Verschierlenes darstellten.

Diese Srhutzworte mußten immer bereit sein; sie gingen im Laufe von etwa 10 Jahren ganz ins Gefühl äber; die Emptindungen, die durch die Schutzworte erzeugt wurden, hatten sich sozusagen akkumuliert, so daß nicht jedesmal neu etwas zu denken war, aber bei besonderen Schwankungen mußten sie auch dann noch und müssen heute nọch in etwas veränderter Form angewandt werden.

Etwa zwei Jahre nach dieser ereignisvollen Weihnachtszeit war ein ziemlicher (Hleichgewichtszustand crreicht. Dabei zeigte sich als Überhleibsel dic all. mähliche Ausbildung bestimmter Wahrnehmungsfähigkeiten und cine Art Kontrolle über den eigenen Körper.

Ich führe der Reihe nach einiges an.

1. Das Vermögen, die Gestalten jederzeit auf Wunsch sehen zu können, sie zu studieren, aber sie nicht gerade sehen zu müssen. (Nach besonderen körperlishen und seelischen Störungen kamen sie allerdings von selhst und wurden gefährlich.)

2. Bei Betrachtung anderer Menschen Gestalten \%ı sehen, die ich für symbolische Ausdrucksformen ihres ('harakters hielt: z. B. pin Mensch in Begleitung eines riesenhaften Polyps, ein anderer mit einer Schlange, die sich um sein Bein herumwand und mit dem Kopf sich drohend gegen dessen Gesicht wandte. Ferner wurden allerlei Farben in der Umgebung von Menschen urd Tieren gesehen. Diese Beobachtungen verliefen folgendermaßen: Konzentrierung der Gedanken auf das Objekt, wobei versucht wurde, dic sinnlich wahrnehmbare Erscheinung desselben hinwegzusuggerieren. Gelang dies, so tauchte dafür die andere Gestalt auf. 
3. Ein Mitempfinden des Gesundheitszustandes anderer. In der Nähe kranker Menschen fühlte ich deren Krankheit am eigenen Körper. Auch bloße Anlagen zu Krankheiten fühlte ich, wenn ich mit den Betreffenden einige Zeit zusammen war. Oft tauchte auch ein Bild der Krankheit auf. Ich sah die kranken Körperteile schwarz bzw. als Hohlräume. Die übertragenen Krankheitsgefühle muBte ich dann durch Schutzworte bekämpfen.

4. Es schien die Regulation des eigenen Körperhaushaltes becinflußt werden zu können.

Krankheiten, Erkältungen, Attacken auf den eigenen Körper eigten sich mir sogleich oder schon vorker in Gestalten typischer Art an, die alle einen düsteren, erschreckenden Charakter trugen. Diese Gestalten konnte ich aber durch meine Schutzworte resp. seelischen Abwehrkräfte verdrängen, aushungern, verdorren lassen, direkt auflösen. Mit diesem Vorgang verschwanden dann auch körperliche Symptome von Unbehagen, und Schwankungen wurden reguliert. Besonders Verdauungsbeschwerden wurden auf diese Weise leicht beeinflußt, unter denen jch vorher sehr zu leiden hatte. Infektionen, Erkältungen gab es nur noch selten.

Die Wirkung der Schutzworte brachte eigenartige körperliche Veränderungen hervor. Nach etwa 10 Minuten langer Anwendung der Schutzworte trat starke Urinabsonderung ein, der Magen wurde leer, die Peristaltik geregelt, ich hatte Organgefühle in den Augen, hinter der Stirn, in der Gegend der Schilddrüse, hinter der Magengegend, ein den ganzen Körper durchrieselndes Gefühl der Lebendigkeit, das in einem Ausstrahlungsgefühl in Händen und Füßen gipfelte. Die Atemzïge wurden sehr verlangsamt. (Später machten sich diese körperlichen Vorgänge in etwas anderer Weise geltend.)

Der Einfluß auf den Körper war nicht unumschränkt. denn der eigene Feind von früher lebte noch und war stets auf der Lauer. Es durften keine ihm günstigen Umstände geschaffen werden. Ich konnte wohl die Gestalten einschrumpfen lassen durch die Schutzworte, aber allzugroße Bemühung und Anstrengung war auch nachteilig, machte sie wieder stark. Ich war auch noch für lange Jahre an strenge Lebensregeln gebunden: z. B. Alkohol vermeiden, nur wenig Fleischnahrung aufnehmen, keine starken Reizmittel nehmen, keine Arzneimittel, fortgesetzte Beherrschung des Gemüts, keine körperlichen Anstrengungen; ich durfte keine Feinde haben, ich sollte kein schlechtes Gewissen haben.

5. Telepathische Fähigkeiten.

Der Zustand von Personen, die mir seelisch nahe standen. räumlich entfernt waren, kündigte sich mir in Form von Gestalten an, oft auch nur durch ein be. sonderes Gefühl. Besondere Gefahren zeigten sich durch ein Schreckbild an, auch Krankheit. Solche Fälle sind mir viele bekannt: ich gebe hier ein Beispiel: Ich sitze zu Hause auf meinem Zimmer und lese. Plötzlich sehe ich eine kekannte Person in verzerrter Gestalt oder von Gestalten drohender Art umgeben, oder symbolisch vor einem Abgrund stehen. In Wirklichkeit war diese Person, wie sich herausstellte, in dieser Zeit in Gefahr, von einem Auto überfahren zu werden.

Später konnte ich auch nach Willkür mich mit Bekannten in Verbindung setzen. Ich konzentrierte meine Gedanken auf eine Person in der Ferne. Zunächst sah ich mich in einem Raume, der angefüllt war mit Gestalten, die sich alle auf mich selbst bezogen (mir meine Gewohnheiten, Charaktereigenschaften, Wünsche, Erlebnisse objektiv andeuten). Die Gestalten brachte ich zum Verschwinden durch die inneren Schutzworte. Die Gestalten schrumpften ein, es entstand ein leerer Raum; die Konzentration in die Ferne wurde wiederaufgenommen unter scharfer Vorstellung des.Gesuchten. Nach einigen Minuten tauchten neue Gestalten auf, auch Gefühle, die mit dem seelischen und körperlichen Zustand des Betreffenden zusammenhingen, wie aus der Kontrolle hinterher hervorging. Bei seelisch Nahestehenden und Blutsverwandten gelang dies am besten. 
Im Laufe der Zeit ließen alle diese Fähigkeiten nach, besonders als ich mit meinem Studium begonnen hatte. Es schob sich gewissermaßen eine Wand ein zwischen meinem Ich und der Welt der innerlichen Wabrnehmungen; ich habe aber die Exfahrung gemacht, daß diese Kulisse durch geeignete Umstände wieder hinweggeschoben werden kann.

Etwas blieb immer und ständig gleich bis zum heutigen Tag; nämlich, ich fühlte und sah um mich herum in einer Entfernung von 3-4 m einen Ringwall, bestehend aus einer mir feindlichen, stets wogenden Substanz, aus der unter gewissen Bedingungen Dämonen wieder hervorbrechen können. Anfangs fiel es mir sehr schwor, den Vorlesungen zu folgen, da ich meine Aufmerksamkeit ständig durch Schutzworte unterbrechen mußte. Auch war das Einprägen ins Gedächtnis außerordentlich schwierig. Ich konnte mir nur mit Hilfe selbstgeschaffener Bilder etwas merken, das alte Ich mit seinem Gedächtnis war in dieser Beziehung sehr untätig gewordèn. Ich brauchte einen besonderen Willensakt zum Einprägen. Letzterer schien vom neuen Ich auszugehen, das ungetrübt erhalten blieb.

\section{Rückblick auf meine Erkrankung.}

Ich kann folgende Etappen im Verlauf der Krankheit abgrenzen, die allerdings nicht ganz scharf ineinander übergingen, mit Ausnahme des 23. Dez. 1901 wo ein plötzlicher Umscḥwung stattfand.

1. Allmähliche Herabdämmerung des Bewußtseins. Beginn im Sommer 1901.

2. Erstes Auftreten der verfolgenden Elemente im Oktober 1901.

3. Rapider Absturz in das Reich der Dämonen. Die seelische Widerstands. kraft droht zusammenzubrechen. Mitte Dezember 1901.

4. Tiefpunkt und Wendung. Neues Jch. Ruckartige Regulation sämtlicher Störungen. 23. Dez. 1901.

5. Allmähliche Erholung. Der Feind bleibt in der Nähe. Schutzworte. Zeitweise Rückfälle. Ende Dez. 1901 und ferner.

6. Allmähliche Emanzipation der Wahrnehmungen vom Zwang. Beziehung derselben zu allen Gebieten des Lebens. Schutz durch das neue Ich und Schutzworte. April 1902 und weiterhin."

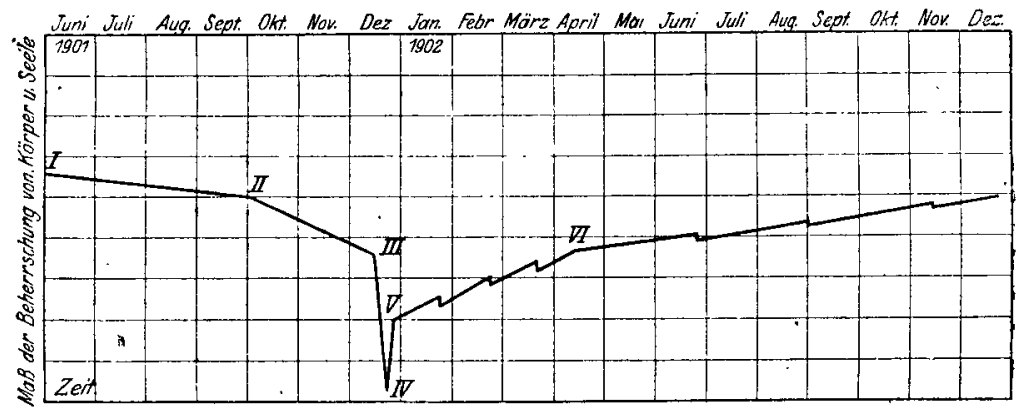

Abb. 1.

Die eigene Auffassung des Patienten von seiner Krankheit.

Der Patient berichtet darüber folgendes:

„Ich glaube, daß ich die Krankheit selbst hervorgerufen habe. Bei dem Versuch, in eine jenseitige Welt einzudringen, stieß ich auf deren natürliche Wächter, die Verkörperungen meiner eigenen Schwächen und Fehler. Ich hielt diese Dämonen anfangs für niedere Bewohner einer jenseitigen Welt, die mich zum Spiel- 
ball benützen konnten, weil ich mich unvorbereitet in diese Regionen begab und dort verirrte. Später hielt ich sie für abgespaltene Teile meines Geistes (Leidenschaftsformen), die im freien Raum in meiner Nähe existierten, sich von meinen Gefühlen ernährten. Ich glaubte, daß sie jeder andere Mensch auch besitze, sie aber durch den Schutz und glücklichen Betrug des persönlichen Existenzgefühls nicht wahrnimmt. Letzteres fasse ich auf als ein Kunstprodukt aus Erinnerungen, Gedankenkomplexen usw., eine nach außen schön vergoldete Puppe, in der nichts Wesentliches lebt.

Bei mir war dieses persönliche Ich porös gemacht durch meine Bewußtseinsherabdämmerungen. Ich wollte mich dadurch einer höheren Lebensquelle näherbringen. Ich hätte zur Vorbereitung vorher lange Zeit hindurch ein höheres überpersönliches Selbst in mir zur Erweckung bringen müssen, denn ,Götterspeise war nichts für sterbliche Lippen. sie wirkte zerstörend auf das, tiermenschliche Selbst, zerspaltete es in seine Teile; diese bröckelten allmählich auseinander, die Puppe wurde geradezu maceriert, der Körper geschädigt. Ich hatte zu früh den Zugang zu den ,Lebensquellen' erzwungen, der Fluch der ,Götter'-kam auf mich herab. Spät erst erkannte ich, daß trübe Elemente sich mitbeteiligt hatten, ich lernte sie kennien, nachdem sie zu große Mracht schon,hatten. Es gab keine Rettung mehr; jetzt hatte ich die Geisterwelt. die ich zu sehen wünschtr. Die Dämonen stiegen ans dem Abgrund auf als die Hüter, als die Zerberusse, die keinen Unbefugten hereinlassen. Ich entschloß mich, den Kampf auf Leben und Tod aufzunehmen. Für mich bedeutete es zuletzt einen Entschluß zu sterben, denn nach meiner Meinung mußte ich alles hinwegtun, was den Feinrl erhält, aber dies war zugleich auch das, was das Leben erhält. Ich wollte in den Tod ohne wahnsimnig zu werden. stand nun sozusagen der Sphinx gegenüber: Fntweder du in den Abgrund oder ich!

In diesem Moment kam die Erleuchtung, ich durchschaute die wahre Natur meiner Verführer durch die Enthaltung von Nahrung. Sie waren Zuhälter und zugleich Betrüger meines lieben persönlichen Ich, das mir jetzt ebenso nichtig wie sie vorkam. Ind indem dann ein größeres umfassenderes Ich auftauchte, war ich imstande, die bisherige Persönlichkeit mit ihrem gesamten Anhang aufzugeben. Ich sah, daß nicht diese bisherige Persönlichkeit die übersinnlichen Reiche betreten kann. Ein furchtbarer Schmerz. gleich dem eines Vernichtungsschlages war die Folge, aber ich war gerettet, die Dämonen schrumpften ein. vergingen, starben. Für mich begann ein völlig neues Leben, ich fühlte mich ron da an anders als andere Menschen. Fin Ich wic sie es haben, bestehend alws konventio: nellen Lügen. Schein, Selbstbetrug, Erinnerungsbildern hat sich bei mir auch wieder gebildet, abre dahinter und darüber stand stets ein größeres umfassencleres Ich, das mir den Findruck des Ewigen. Unveränderlichen, Unsterblichen, Unbefleckbaren macht, das seitdem mein Schutz und stets meine Zuflucht gewesen ist. Ich glaube, diı es für viele Menschen von Vorteil wäre, wenn sie ein solehes höheres Ich kennen würden, daß es Menschen gibt, die anf günstigeren Wegen zu einem solchen tatsächlich gekommen sind."

\section{Phänomenologische Analyse.}

Die Diagnose ist jedem psychiatrisch sachverstândigen Leser selbstverständlich. Es handelt sich in psychopathologischer Beziehung um eine schizophrene Veränderung des Seelenlebens. Obgleich der Fall durch sich selbst klar ist, erscheint es mir sinnvoll, einige phänomenologische Hauptpunkte anschließend hervorzuheben. 
a) BewuBtseinszustand.

Bei Betrachtung des Bewußtseins können wir feststellen, daß dasselbe im allgemeinen stets klar war. Bewußtseinstrübungen mit Verwirrtheitszustand und Desorientierung traten für Augenblicke auf wie beim Gesunden kur\% nach Erwachen aus einem lebhaften Traum; z. B. der Patient erwachte nachts, fand sich umringt von seinen Wahngebilden, mußte aufstehen, im Zimmer umhergehen, um sich zu vergewissern, wo er ist. Während der halluzinatorischen Wahrnehmungen selbst kam es zu vorübergehenden Delirien; der Patient fühlte sich mit seinen Dämonen im selben Raum, war entrückt.

Dämmerzustände traten nicht auf, obwohl man von vorübergehenden Bewußtseinseinengungen reden kann. Der Gansersche Symptomenkomplex ist nicht nachzuweisen.

\section{b) Persönlichkeitsbewußtsein.}

Eine Spaltung des Bewußtseins (alternierendes Bewußtsein) liegt hier nicht vor. Patient spricht wohl von einem zweiten Ich, aber nicht ron zwei Inhalten. Das zweite ist nicht neben dem ersten vorhanden, sondern demselben übergeordnet.

Die Verkleinerung des Bewußtseins, von der Patient spricht, ist nicht als Ekmmesie aufzufassen; er fühlte sich wie ein ,neugeborenes Kind", war jedoch nicht in eine frühere Periode seines Lebens "zurïckversetzt. Man könnte hier an Kleinheitswahn denken. Hervorzuheben ist das Gefühl der Veründerung der ganzen Persönlichkeit, das den schizophrenen ('harakter der Psychose zeigt.

Patient glaubt zu einem mathematischen Punkt zu verschwinden, sein Ich ganz zu verlieren, er fühlt sich aufgeteilt in die Gegenstände der Umwelt; oder er steht in Gefahr, ,blödsinnig." zu werden, muß alles aufbieten, $11 \mathrm{~m}$ nicht von einem, Dämon besessen zu werden".

\section{c) Ablauf des Denkens.}

Bei der Betrachtung des Gedankenablanfes finden wir hier Zustände c̄er Tenkhemmung, z. B. die Unmöglichkeit, einen Brief zu schreiben, Gesprochenes anzuhören. Die zu rasche Ermüdung und schlechte Konzentrationsfähigkeit ist als deren Ursache anzusehen. Ideenflucht liegt nicht vor, auch keine Spaltung 'zwischen Denken und Fühlen. Im Vordergrund des Erlebens des Patienten stehen die inhaltlichen Störungen des Gedankenablaufes, es handelt sich um Gesichts- und Ge. hörshalluzinationen. Patient verlegt seine Halluzinationen weder in den äußeren noch in den inneren Raum, sondern erfindet einen besonderen dritten Raum. Offenbar sind es Pseudohalluzinationen, zum Teil Traumhalluzinationen, die sich aber nach seinen Angabon noch 
mehr von normalen Vorstellungen unterscheiden als gewöhnliche Pseudohalluzinationen. Er legt im letzten Teil seiner Beschreibung eine gewisse transzendente Beziehung dieser halluzinatorischen Gebilde zur Umwelt, zum wirklichen Geschehen nahe. So besteht bei ihm relativ positives Realitätsurteil. Er denkt in seinen ,Wahrnehmungen“ nach der Psychose an telepathische Eindrücke, die sich im Bewußtsein in Bilder und Töne umwandeln. Wie die Wirkung seiner ,Schutzworte" auf gewisse Punkte des Körpers, die anscheinend innersekretorische Organe oder sympathische Ganglien decken, zu erklären ist, ist dunkel, wenn man sie nicht als kinästhetische Halluzination auffassen will.

Die Wahnideen, die .Patient hatte, sind nicht systematisiert; er hatte den Glauben, daß er seine „Dämonen“ durch Eindringenwollen in eine übersinnliche Welt heraufbeschworen habe, indem er nicht die

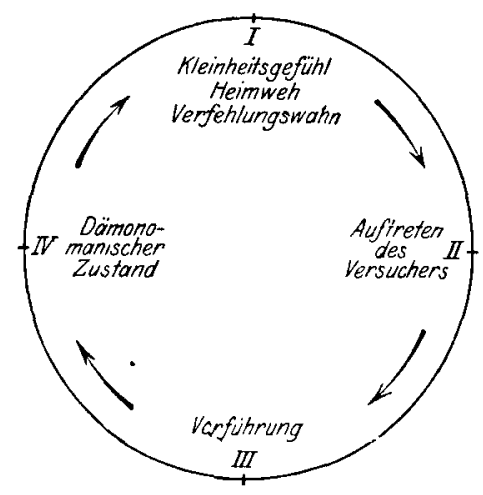

Abb. 2. nötige Vorbereitung gehabt habe, die von dort kommenden Angriffe auszuhalten. Dazu tritt dann ein Kleinheitswahn und Verfehlungswahn. Es ist noch das Eigenartige zu konstatieren, daß die Gestalten nicht nur durch ihre gesprochenen Worte, sondern auch durch ihr Aussehen und Auftreten seine Schwächen und Unvollkommenheiten anzeigen; sie sind ihm ein Echo seiner eigenen Natur. Nicht minder zu beachten ist das „Zusammenschrumpfen der Dämonen" bei Anwendung der Gegenaffekte. Letztere sind nicht Ausdruck von Größenideen selbst, wenn Patient sagt ,ich bin das Absolute". Ich hebe noch den Zyklus im Höhestadium der Psychose hervor. Der „Logiker", der in ihm spricht, ist ein typisches Beispiel ,,gemachter Gedanken" und zeigt deutlich wieder die schizophrene Natur der Psychose an. Vorausgesetzt, daß die Beobachtungen des Patienten richtig sind, könnte man diesen Zyklus unter die Rubrik der ekstatischen Erlebnisse der Mönche und Mystiker und ihrer Prüfungen zählen.

Der Glaube des Patienten an Dämonen läßt daran denken, daß dieser Zyklus ein System eines physikalischen Verfolgungswahnes ist. Err verhält sich aber, seinen eigenen Erlebnissen gegenüber, höchst beobachtend, forschend und findet dadurch schließlich den Ausweg aus dem Labyrinth. Auch die Empfindung, daß er verloren sei, kann nicht aus einem hypochondrischen Wahn, unheilbar zu sein, hervorgegangen sein, denn dies Urteil bildete er sich erst, nachdem er trotz Kampf sehon wußte, daß er immer mehr in das Labyrinth seiner dämonischen Welt hineingezogen wurde, wovor er sich nur durch Verweigerung der 
Nahrung schützen konnte, und, nachdem er „,des Pudels Kern" entdeckt hatte, auch den Sieg davon trug. Er wollte übrigens sterben, um wenigstens nicht blödsinnig zu werden.

\section{d) Intellekt.}

Die intellektuellen Fähigkeiten waren nicht getrübt, Gedächtnis und Urteilsfähigkeit scharf, ersteres nach der Psychose mangelhaft, da es, wie Patient glaubt, weniger automatisch arbeitete als früher (ein schizophrenes Zeichen). Für die Merkfähigkeit ist hervorzuheben, daß er Gesprächen zuhören konnte, ohne etwas zu verstehen, jedoch lag dies seinem Willen ob. Während der Psychose selbst durfte er wegen seiner Selbstverteidigung gegen die Dämonen nicht aufpassen, wie er auch in späterer Zeit durch Anwendung seiner Schutzworte Schwierigkeiten bekommt, der Vorlesung zu folgen. Die Unaufmerksamkeit deckte sich nicht mit dem Mangel an Einstellung (Zerfahrenheit) der Hebephrenen und Katatoniker; er konnte aufpassen, hatte nur sozusagen keine Zeit.

\section{e) Gefunls-und Gemütszustände.}

Die Grundstimmung war ängstlich und traurig, bisweilen ratlos. Die Ängstlichkeit steigerte sich bis zur Angsterregung und Verzweiflung. Patient läuft umher, sucht Schutz bei der Mutter, flüchtet sich wieder vor der Mutter. Er findet mehr Ruhe bei gleichmütigen seelenstarken Menschen.

Zornesausbrüche und Wut traten nicht auf als Reaktion auf die Halluzinationen. Patient mußte im Gegenteil alle Affekte zu unterdrücken suchen, da er sonst, wie er meint, den Dämonen wieder Kraft zugeführt hätte. Doch zeigt er ein äußerst empfindliches Wesen und Reizbarkeit, indem er durch die geringste Mißbilligung von seiten seiner Umgebung, oder schon durch bloßes Zureden in Unruhe versetzt wurde, die seine „Dämonen" herbeilockte. Er mußte sich der Ruhe und Affektlosigkeit befleißigen, um das seelische Gleichgewicht zu behalten. Dazu erfindet er seine "Schutzworte“", die als Gegenaffekte aufzufassen sind; er will das Denken von allem Geformten und Differenzierten abziehen, um dadurch über die krankhaften Vorstellungen Herr zu werden.

Hervorzuheben sind abnorme Gefühlszustände. Zuerst tauchen ekstatische Freiheitsgefühle auf, Gefühle der Wonne des Ausgeglichenseins, als er, angeregt durch vitalistische Vorstellungen, sich auf das „Absolute" konzentrierte.

Später erlebt er Schwebegefühl und merkwürdige Sensationen an körperlichen Organen, es wollen durch Sinnesreize die „Dämonen“ 
herein. Typisch sind in der Zeit der „Entscheidung" die Gefühle des Zerrissenwerdens, des Ausdorrens, des Hungers, Verbanntseins, der Öde, des Hinsterbens.

\section{f) Willenss phäre.}

Auf dem Gebiet der Willenssphäre zeigt sich eine subjektive Insuffizienz. Patient sitzt da, glaubt nichts tun zu können. Im Berufe bleibt (r) untätig; soll er Briefe schreiben, so legt er die Feder wieder weg. Zwar gibt er an, er sei nur deshalb gehemmt gewesen etwas zu tun, weil er ständig aufpassen mußte, daß der „Feind" nicht näher komme. Vielleicht lassen diese Angaben auf einen Pseudostupor schließen. Motorische Unruhe entsprang bei dem Patienten stets der Angst. Das Kraftgefühl, das während und nach der Úbung der „Schutzworte" entstand, ist noch hervorzuheben. Er kennt dies Gefühl heute noch und beschreibt es als ausgehend von der Magengrube nach der Wirbelsäule hinziehend, damn durch die Extremitäten ausstrahlend, ohne die Muskeln zu spannen, zugleich auch nach allen Seiten die Haut des Körpers durchdringend.

\section{Literaturverzeichnis.}

1. Jaspers, Die phänomenologische Forschungsrichtung in der Psychopathologie. Zeitschr. f. d. ges. Neur. u. Psych. 9, Anmerk. S. 398. 1912.

2. Gruhle, H. W., Selbstschilderung und Einfühlung. Zugleich ein Versuch der Analyse des Falles Benting. Zeitschr. f. d. ges. Neur. u. Psych. 28, 148. 1915.

3. Joh. Müller, Über die phantastischen Gesichtserscheinungen. Koblenz 1816, S. 20. Zitat im Auszug bei Jaspers: Psychopathologie.

4. Kandinsky, Kritik und klinische Betrachtung im Gebiete der Sinnestäuschungen. Berlin 1885. Archiv f. Psych. 11, 453.

5. Kieser, Melancholia daemonomaniaca occulta. Allg. Zeitschr. f. Psych. 10, 423.1853.

6. Fngelken, Selbstbericht cines Geisteskranken nebst Krankheitsgeschichte und Bemerkungen. Allg. Zeitschr. f. Psych. 6, 586. 1849.

7. Th. de Quincey, Bekenntnisse eines Opiumessers. Deutsch. Stuttgart 1886.

8. Schreber, Denkwürdigkeiten eines Nervenkranken. Leipzig 1903.

9. Fehrlin, Die Schizophrenie. Selbstverlag 1910.

10. James, Die religiöse Frfahrung in ihrer Mannigfaltigkeit. Deutsch. Leipzig 1907.

11. Forel, Selbstbiographie eines Falles von Mania acuta. Archiv f. Psych. 34,960 .

12. Jaspers, Kausale und verständliche Zusammenhänge zwischen Schicksal und Psychose bei Dem. praecox. 